PROCEEDINGS OF THE

AMERICAN MATHEMATICAL SOCIETY

Volume 138, Number 4, April 2010, Pages 1511-1515

S 0002-9939(09)10165-X

Article electronically published on November 12, 2009

\title{
FIXED POINTS IN INDECOMPOSABLE $k$-JUNCTIONED TREE-LIKE CONTINUA
}

\author{
CHARLES L. HAGOPIAN
}

(Communicated by Alexander N. Dranishnikov)

\begin{abstract}
Let $M$ be an indecomposable $k$-junctioned tree-like continuum. Let $f$ be a map of $M$ that sends each composant of $M$ into itself. Using an argument of $\mathrm{O}$. H. Hamilton, we prove that $f$ has a fixed point.
\end{abstract}

D. P. Bellamy [1] in 1979 defined a tree-like continuum that does not have the fixed-point property (also see [4, 10], [11, and [13]). In 1998, the author [6] proved that every tree-like continuum has the fixed-point property for deformations. This was accomplished by showing that every map of a tree-like continuum $M$ that sends each arc-component of $M$ into itself has a fixed point. The collection of arc-components is a partition of a continuum. Hence a map that sends each arc-component into itself is sometimes called an arc-component-preserving map. Bellamy's continuum is indecomposable and its arc-components are composants. The collection of composants of an indecomposable continuum is a partition that is refined by the collection of arc-components. The difference between these two partitions is sometimes extreme. Each arc-component consists of one point in P. Minc's example [11] of a hereditarily indecomposable tree-like continuum without the fixed-point property. Must every composant-preserving map of an indecomposable tree-like continuum $M$ have a fixed point? We prove the answer is yes if $M$ is $k$-junctioned.

\section{Preliminaries}

A space $X$ has the fixed-point property if for each map $f$ of $X$ into $X$ there is a point $x$ of $X$ such that $f(x)=x$.

A chain is a finite collection $\mathcal{A}=\left\{A_{1}, A_{2}, \ldots, A_{n}\right\}$ of open sets such that $A_{i} \cap A_{j} \neq$ $\emptyset$ if and only if $|i-j| \leq 1$. The elements $A_{1}$ and $A_{n}$ are called end links of $\mathcal{A}$. Each element of $\mathcal{A} \backslash\left\{A_{1}, A_{n}\right\}$ is called an interior link of $\mathcal{A}$. If $n>2$ and $A_{1}$ also intersects $A_{n}$, the collection $\mathcal{A}$ is called a circular chain.

A collection $\mathcal{E}$ of sets is coherent if for each nonempty proper subcollection $\mathcal{F}$ of $\mathcal{E}$ there is an element of $\mathcal{F}$ that intersects an element of $\mathcal{E} \backslash \mathcal{F}$.

Received by the editors December 4, 2008, and, in revised form, December 22, 2008, and August 1, 2009.

2010 Mathematics Subject Classification. Primary 54F15, 54H25.

Key words and phrases. Fixed point, indecomposable, composant-preserving map, $k$ junctioned, tree-like continuum.

The author wishes to thank Marcus Marsh, Janusz Prajs, and the referee for suggestions that led to the improvement of this paper and Michael Heacock for drawing Figure 1.

(C)2009 American Mathematical Society 
A finite coherent collection $\mathcal{T}$ of open sets is a tree chain if no three elements of $\mathcal{T}$ have a point in common and no subcollection of $\mathcal{T}$ is a circular chain. An element of $\mathcal{T}$ that intersects more than two other elements of $\mathcal{T}$ is called a junction link of $\mathcal{T}$. Let $\mathcal{J}$ be the collection of junction links of $\mathcal{T}$. Each maximal chain in $\mathcal{T} \backslash \mathcal{J}$ is called a chain section of $\mathcal{T}$.

A continuum is a nondegenerate compact connected metric space.

A continuum is indecomposable if it is not the union of two proper subcontinua. A continuum is hereditarily indecomposable if each of its subcontinua is indecomposable.

Let $x$ be a point of a continuum $M$. The $x$-arc-component of $M$ is the union of $\{x\}$ and all arcs in $M$ that contain $x$. The $x$-composant of $M$ is the union of all proper subcontinua of $M$ that contain $x$. If $M$ is indecomposable, then $M$ is the union of uncountably many dense disjoint composants.

A tree chain with mesh less than a number $\epsilon$ is called an $\epsilon$-tree-chain.

A continuum $M$ is tree-like if for each positive number $\epsilon$ there is an $\epsilon$-tree-chain covering $M$.

A tree-like continuum $M$ is $k$-junctioned if $k$ is the least integer such that for every positive number $\epsilon$ there is an $\epsilon$-tree-chain covering $M$ with $k$ junction links, [3], [5].

A tree-like continuum $M$ has the chain-section property if for each positive number $\epsilon$ there is an $\epsilon$-tree-chain $\mathcal{T}$ covering $M$ that has a chain section $\mathcal{S}$ such that each point of $M$ is within the distance $\epsilon$ of an element of $\mathcal{S}$.

\section{RESUlts}

Theorem 2.1. Suppose that $f$ is a map of an indecomposable tree-like continuum $M$ that sends each composant of $M$ into itself. Suppose that $M$ has the chain-section property. Then $f$ has a fixed point.

Proof. Assume that $f$ moves each point of $M$. Let $\rho$ be a metric on $M$. Let $\epsilon$ be a positive number such that $\rho(x, f(x))>\epsilon$ for each point $x$ of $M$.

Let $\left\{\Sigma_{i}: i=1,2, \ldots\right\}$ be a countable open base for $M$. For each positive integer $i$, let $\Omega_{i}=\left\{x \in M: x\right.$ and $f(x)$ belong to a continuum in $\left.M \backslash \Sigma_{i}\right\}$. Each $\Omega_{i}$ is a closed subset of $M$ [12, Th.58, p.23] and $M=\bigcup\left\{\Omega_{i}: i=1,2, \ldots\right\}$ since $f$ sends each composant into itself. By the Baire Category Theorem, there is an integer $j$ such that $\Omega_{j}$ contains a nonempty open subset $E$ of $M$. Let $F$ be a nonempty open subset of $M$ whose closure is contained in $E$.

Since $M$ is indecomposable, the open set $\Sigma_{j}$ cannot be contained in any single component of $M \backslash F$. Hence $M \backslash F$ is the union of two separated sets $V$ and $W$ that intersect $\Sigma_{j}\left[12\right.$, Th. 44, p. 15]. Let $v$ and $w$ be points of $V \cap \Sigma_{j}$ and $W \cap \Sigma_{j}$, respectively. Let $\epsilon_{1}$ be a positive number less than $\epsilon, \rho(V, W), \frac{1}{3} \rho\left(\{v, w\}, M \backslash \Sigma_{j}\right)$, and $\rho(F, M \backslash E)$.

Let $\mathcal{T}_{1}$ be an $\epsilon_{1}$-tree-chain $\mathcal{T}_{1}$ covering $M$ with a chain section $\mathcal{S}_{1}$ such that each point of $M$ is within $\epsilon_{1}$ of an element of $\mathcal{S}_{1}$. Let $\mathcal{A}=\left\{A_{1}, A_{2}, \ldots, A_{n_{1}}\right\}$ be a chain consisting of interior links of $\mathcal{S}_{1}$ such that $\rho\left(v, A_{1}\right)<\epsilon_{1}$ and $\rho\left(w, A_{n_{1}}\right)<\epsilon_{1}$. Note that $A_{1} \subset V \cap \Sigma_{j}$ and $A_{n_{1}} \subset W \cap \Sigma_{j}$. Since $F$ separates $A_{1}$ from $A_{n_{1}}$ in $M$, there is an interior link $A_{i_{1}}$ of $\mathcal{A}$ that intersects $F$. Note that $A_{i_{1}} \subset E \subset \Omega_{j}$.

By the uniform continuity of $f$, there is a positive number $\delta$ such that for each subset $X$ of $M$ having diameter less than $\delta$, both $X$ and $f(X)$ are contained in elements of $\mathcal{T}_{1}$. 
Let $\hat{v}$ and $\hat{w}$ be points of $A_{1}$ and $A_{n_{1}}$, respectively.

Let $\epsilon_{2}$ be a positive number less than $\epsilon_{1}, \delta, \frac{1}{2} \rho\left(\hat{v}, M \backslash A_{1}\right)$, and $\frac{1}{2} \rho\left(\hat{w}, M \backslash A_{n_{1}}\right)$.

Let $\mathcal{T}_{2}$ be an $\epsilon_{2}$-tree-chain covering $M$ that has a chain section $\mathcal{S}_{2}$ such that each point of $M$ is within $\epsilon_{2}$ of an element of $\mathcal{S}_{2}$. Since no subcollection of $\mathcal{T}_{1}$ is a circular chain, there exists a subchain $\mathcal{B}=\left\{B_{1}, B_{2}, \ldots, B_{n_{2}}\right\}$ of $\mathcal{S}_{2}$ such that $\mathcal{B}$ runs through $\mathcal{A}$ (that is, $B_{1} \subset A_{1}, B_{n_{2}} \subset A_{n_{1}}$, and $\cup \mathcal{B} \subset \cup \mathcal{A}$ ). Let $B_{i_{2}}$ be the first link of $\mathcal{B}$ contained in $A_{i_{1}}$ and let $B_{j_{2}}$ be the last link of $\mathcal{B}$ contained in $A_{i_{1}}$.

Since $M$ is indecomposable, $M \backslash B_{1}$ is the union of separated sets $Y$ and $Z$ that intersect $B_{n_{2}}$. Let $y$ and $z$ be points of $Y \cap B_{n_{2}}$ and $Z \cap B_{n_{2}}$, respectively. Let $\epsilon_{3}$ be a positive number less than $\epsilon_{2}, \rho(Y, Z)$, and $\frac{1}{3} \rho\left(\{y, z\}, M \backslash B_{n_{2}}\right)$.

Let $\mathcal{T}_{3}$ be an $\epsilon_{3}$-tree-chain covering $M$ that refines $\mathcal{T}_{2}$ and has a chain section $\mathcal{S}_{3}$ such that each point of $M$ is within $\epsilon_{3}$ of an element of $\mathcal{S}_{3}$. Let $\mathcal{C}=\left\{C_{1}, C_{2}, \ldots, C_{n_{3}}\right\}$ be a chain of interior links of $\mathcal{S}_{3}$ such that $C_{1} \subset Y \cap B_{n_{2}}$ and $C_{n_{3}} \subset Z \cap B_{n_{2}}$. Since $\mathcal{T}_{2}$ does not contain a circular chain and $B_{1}$ separates $Y$ from $Z$ and hence $C_{1}$ from $C_{n_{3}}$ in $M$, there exist two subchains of $\mathcal{C}$ such that one subchain runs through the chain $\left\{Y \cap B_{n_{2}}, Y \cap B_{n_{2}-1}, \ldots, Y \cap B_{2}, B_{1}\right\}$ and is followed by the second that runs through $\left\{B_{1}, Z \cap B_{2}, \ldots, Z \cap B_{n_{2}}\right\}$.

Since $\mathcal{C}$ is comprised of interior links, the collection $\mathcal{T}_{3} \backslash \mathcal{C}$ consists of two coherent subcollections $\mathcal{D}$ and $\mathcal{E}$ such that $\bigcup \mathcal{D} \cap \bigcup \mathcal{E}=\emptyset, C_{1} \cap \bigcup \mathcal{D} \neq \emptyset$, and $C_{n_{3}} \cap \bigcup \mathcal{E} \neq \emptyset$.

Let $C_{0}=\bigcup \mathcal{D}$ and $C_{n_{3}+1}=\bigcup \mathcal{E}$. Let $\mathcal{F}$ denote the chain $\left\{C_{0}, C_{1}, \ldots, C_{n_{3}}, C_{n_{3}+1}\right\}$.

Let $J$ be a subcontinuum of $M$ in $\bigcup \mathcal{C}$ that intersects both $C_{1}$ and $C_{n_{3}}$ [12, Th. 44, p. 15].

Let $K=\{x \in J$ : a link of $\mathcal{F}$ containing $x$ follows a link of $\mathcal{F}$ containing $f(x)\}$.

Let $L=\{x \in J$ : a link of $\mathcal{F}$ containing $x$ precedes a link of $\mathcal{F}$ containing $f(x)\}$.

Since $\epsilon_{3}<\epsilon, J \subset \cup \mathcal{C}$, and $f$ is continuous, it follows that $K$ and $L$ are disjoint closed subsets of $J$ and $J=K \cup L$ [8]. Note that since $J$ is connected, either $K$ or $L$ must be empty.

Since $B_{i_{2}} \subset \Omega_{j}$ and $A_{1} \cup A_{n_{1}} \subset \Sigma_{j}$, for each point $x$ of $B_{i_{2}}$ there is a continuum in $M \backslash\left(A_{1} \cup A_{n_{1}}\right)$ that contains $x$ and $f(x)$. Consequently, since $\epsilon_{2}<\delta$, it follows that $f\left(B_{i_{2}}\right)$ is contained in an interior link $A_{j_{1}}$ of $\mathcal{A}$.

Furthermore, $i_{1} \neq j_{1}$ since $\epsilon_{1}<\epsilon$.

Note $i_{1}>j_{1}$. To see this, suppose $i_{1}<j_{1}$. Let $C_{\alpha}$ be the last link of $\mathcal{F}$ that is contained in $Y \cap B_{i_{2}}$. Let $C_{\gamma}$ be the first link of $\mathcal{F}$ that follows $C_{\alpha}$ and lies in $B_{1}$. Note $\bigcup\left\{C_{\alpha}, C_{\alpha+1}, \ldots, C_{\gamma}\right\} \subset \bigcup\left\{B_{1}, B_{2}, \ldots, B_{i_{2}}\right\}$. Since $B_{i_{2}}$ is the first link of $\mathcal{B}$ in $A_{i_{1}}$, it follows that $A_{j_{1}} \cap \bigcup\left\{C_{\alpha}, C_{\alpha+1}, \ldots, C_{\gamma}\right\}=\emptyset$. Note $C_{1} \cup C_{\gamma} \subset \Sigma_{j}$, $C_{\alpha} \subset \Omega_{j}$, and $f\left(C_{\alpha}\right) \subset A_{j_{1}}$. Let $a$ be a point of $C_{\alpha}$. Then $f(a) \in \bigcup\left\{C_{1}, C_{2}, \ldots, C_{\alpha}\right\}$, for otherwise, every subcontinuum of $M$ that contains $a$ and $f(a)$ intersects $\Sigma_{j}$, which is impossible since $a \in \Omega_{j}$. Therefore $a \in K$. Hence $K$ is not empty. See Figure 1. Larger black/white and colored pictures of Figure 1 are posted on http://webpages.csus.edu/ hagopian.

Let $C_{\beta}$ be the first link of $\mathcal{F}$ that lies in $Z \cap B_{i_{2}}$. Let $b$ be a point of $C_{\beta}$. It follows from the argument above that $f(b) \in \bigcup\left\{C_{\beta}, C_{\beta+1}, \ldots, C_{n_{3}}\right\}$. Therefore $b \in L$. Hence $K$ and $L$ are nonempty disjoint closed sets whose union is $J$, and this contradicts the connectivity of $J$. Thus $i_{1}>j_{1}$.

Since $B_{j_{2}}$ is the last link of $\mathcal{B}$ in $A_{i_{1}}$, it follows from the same argument that $f\left(B_{j_{2}}\right)$ is in an interior link $A_{k_{1}}$ of $\mathcal{A}$ and $i_{1}<k_{1}$. 


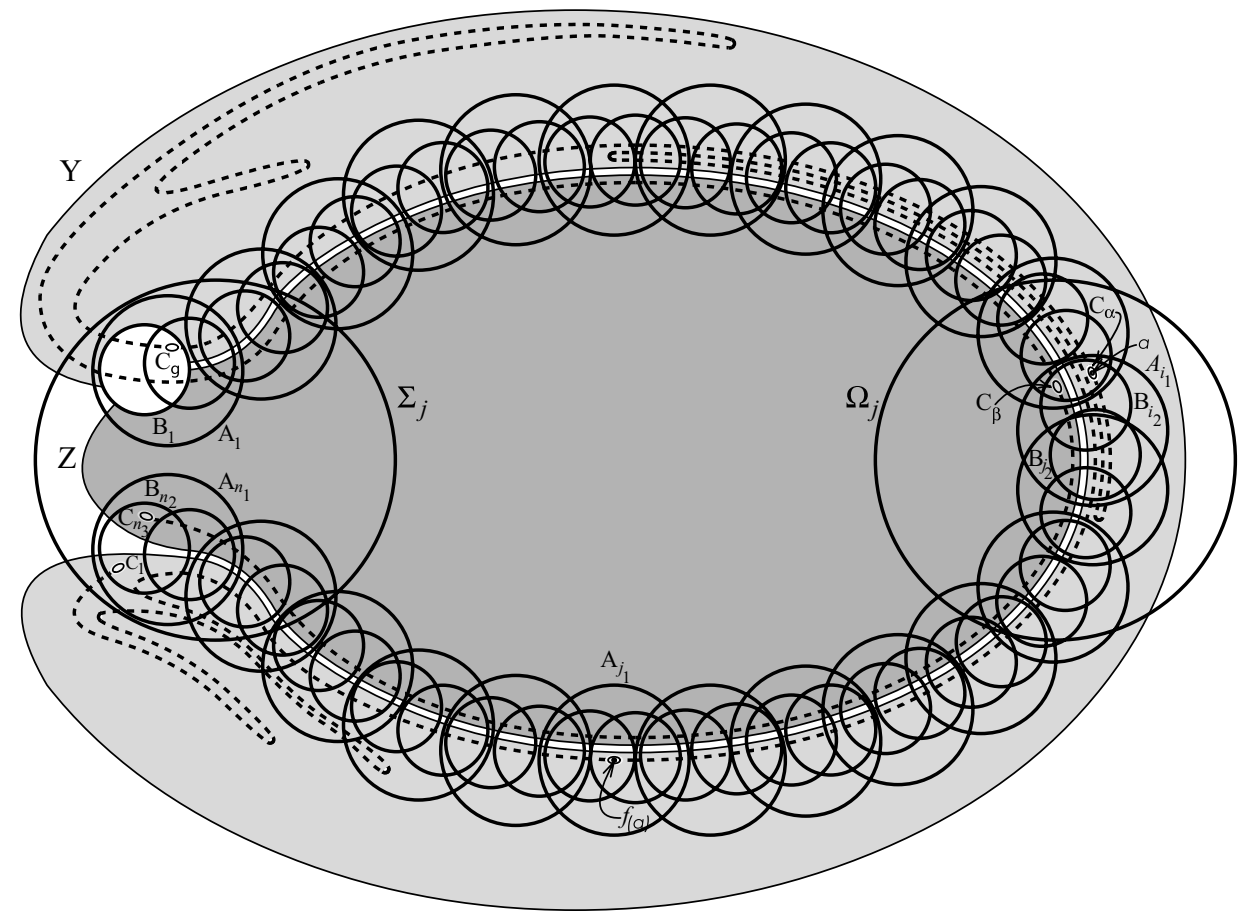

Figure 1

The collection $\mathcal{T}_{1} \backslash \mathcal{A}$ consists of two coherent subcollections $\mathcal{G}$ and $\mathcal{H}$ such that $\bigcup \mathcal{G} \cap \bigcup \mathcal{H}=\emptyset, A_{1} \cap \bigcup \mathcal{G} \neq \emptyset$, and $A_{n_{1}} \cap \bigcup \mathcal{H} \neq \emptyset$. Let $A_{0}=\bigcup \mathcal{G}$ and $A_{n_{1}+1}=\bigcup \mathcal{H}$. Let $\mathcal{K}$ denote the chain $\left\{A_{0}, A_{1}, \ldots, A_{n_{1}}, A_{n_{1}+1}\right\}$.

Let $\mathcal{L}$ denote the chain $\left\{B_{i_{2}}, B_{i_{2}+1}, \ldots, B_{j_{2}}\right\}$. Note $\mathcal{L}=\left\{B_{i_{2}}, B_{j_{2}}\right\}$ in Figure 1 . However, $\mathcal{B}$ may be folded in $\mathcal{A}$, and each interior link of $\mathcal{A}$ may contain a link of $\mathcal{L}$.

Let $P$ be a subcontinuum of $M$ in $\bigcup \mathcal{L}$ that intersects both $B_{i_{2}}$ and $B_{j_{2}}$.

Let $Q=\{x \in P$ : a link of $\mathcal{K}$ containing $x$ follows a link of $\mathcal{K}$ containing $f(x)\}$.

Let $R=\{x \in P$ : a link of $\mathcal{K}$ containing $x$ precedes a link of $\mathcal{K}$ containing $f(x)\}$.

Note $B_{i_{2}} \cup B_{j_{2}} \subset A_{i_{1}}, f\left(B_{i_{2}}\right) \subset A_{j_{1}}, f\left(B_{j_{2}}\right) \subset A_{k_{1}}$, and $j_{1}<i_{1}<k_{1}$. Thus neither $Q$ nor $R$ is empty. Since $\epsilon>\epsilon_{1}, P \subset \cup \mathcal{A}$, and $f$ is continuous, it follows that $Q$ and $R$ are disjoint closed subsets of $P$ and $P=Q \cup R$. This contradiction of the connectivity of $P$ completes the proof.

Theorem 2.2. If $M$ is an indecomposable $k$-junctioned tree-like continuum, then $M$ has the chain-section property.

Proof. Let $\epsilon$ be a given positive number. Let $\mathcal{U}$ be an $\epsilon$-tree-chain covering $M$. Since the composants of $M$ are dense and disjoint, there exists a collection $\mathcal{V}$ consisting of $k+1$ disjoint subcontinua of $M$ such that each element of $\mathcal{V}$ intersects each element of $\mathcal{U}$. Let $\delta$ be a positive number less than $\epsilon$ and the distance between each two elements of $\mathcal{V}$. Let $\mathcal{T}$ be a $\delta$-tree-chain covering $M$ that has only $k$ junction links. Note that no junction link of $\mathcal{T}$ intersects more than one element of $\mathcal{V}$. Since each element of $\mathcal{V}$ is connected, there is a chain section $\mathcal{S}$ of $\mathcal{T}$ that covers an element 
$V$ of $\mathcal{V}$. Since $V$ intersects each element of $\mathcal{U}$, every point of $M$ is within $\epsilon$ of an element of $\mathcal{S}$.

Theorem 2.3. Suppose that $M$ is an indecomposable $k$-junctioned tree-like continuum. Then every composant-preserving map of $M$ has a fixed point.

Proof. Theorem 2.3 follows directly from Theorems 2.1 and 2.2 .

\section{Questions}

Theorem 2.3 provides partial answers to the following open questions.

Question 1. Must every composant-preserving map of an indecomposable tree-like continuum have a fixed point?

Question 2. Does every $k$-junctioned tree-like continuum have the fixed-point property?

Does every plane continuum that does not separate the plane have the fixed-point property? This is the unsolved classic plane fixed-point problem. It is not known if every tree-like continuum in the plane has the fixed-point property. Questions 1 and 2 are also open for tree-like plane continua. For more information and unsolved fixed-point problems, see [2], 7], and [9].

\section{REFERENCES}

[1] D. P. Bellamy, A tree-like continuum without the fixed point property, Houston J. Math. 6 (1980), 1-13. MR575909 (81h:54039)

[2] R. H. Bing, The elusive fixed point property, Amer. Math. Monthly 76 (1969), 119-132. MR 0236908 (38:5201)

[3] C. E. Burgess, Homogeneous continua which are almost chainable, Canadian J. Math. 13 (1961), 519-528. MR0126255 (23:A3551)

[4] L. Fearnley and D. G. Wright, Geometric realization of a Bellamy continuum, Bulletin London Math. Soc. 25 (1993), 177-183. MR1204071 (94b:54095)

[5] C. L. Hagopian, The fixed-point property for almost chainable homogeneous continua, Illinois J. Math. 20 (1976), 650-652. MR0418057 (54:6101)

[6] C. L. Hagopian, The fixed-point property for deformations of tree-like continua, Fundamenta Math. 155 (1998), 161-176. MR1606519 (99b:54046)

[7] C. L. Hagopian, An update on the elusive fixed-point property, Open Problems in Topology. II, edited by E. Pearl, Elsevier B. V. 2007, 263-277.

[8] O. H. Hamilton, A fixed point theorem for pseudo-arcs and certain other metric continua, Proc. Amer. Math. Soc. 2 (1951), 173-174. MR0039993 (12:627f)

[9] R. Mańka, The topological fixed point property-An elementary continuum-theoretic approach, Banach Center Publication, 77, Polish Academy of Sciences, Warsaw, 2007, 183200. MR 2338584 (2008f:54053)

[10] P. Minc, A tree-like continuum admitting fixed point free maps with arbitrarily small trajectories, Topology Appl. 46 (1992), 99-106. MR1184108 (94a:54108)

[11] P. Minc, A hereditarily indecomposable tree-like continuum without the fixed point property, Trans. Amer. Math. Soc. 352 (2000), 643-654. MR1695031 (2000k:54029)

[12] R. L. Moore, Foundations of point set theory, rev. ed., Amer. Math. Soc. Colloq. Publ., 13, Amer. Math. Soc., Providence, RI, 1962. MR0150722 (27:709)

[13] L. G. Oversteegen and J. T. Rogers, Jr., Fixed-point-free maps on tree-like continua, Topology Appl. 13 (1982), 85-95. MR637430 (83b:54044)

Department of Mathematics and Statistics, California State University, Sacramento, 6000 J Street, Sacramento, California 95819

E-mail address: hagopian@csus.edu 